\title{
Species-based comparison of disease severity and risk factors for disseminated Candida infections in pediatric patients
}

This article was published in the following Dove Press journal:

Infection and Drug Resistance

18 April 2016

Number of times this article has been viewed

\section{John J Hawkshead III' \\ Russell B Van Dyke ${ }^{2}$ \\ Susan E Hassig ${ }^{3}$ \\ Larry S Webber ${ }^{4}$ \\ Rodolfo E Begue ${ }^{5}$}

'Merck \& Co, CORE Hospital Specialty Group, Rahway, NJ, ${ }^{2}$ Department of Pediatrics, Tulane University School of Medicine, ${ }^{3}$ Department of Epidemiology,

${ }^{4}$ Department of Biostatistics, Tulane University School of Public Health and Tropical Medicine, ${ }^{5}$ Department of Pediatrics, Louisiana State University Health Sciences Center, New Orleans, LA, USA
Correspondence: John J Hawkshead III Merck \& Co, CORE Hospital Specialty Group, I 26 East Lincoln Avenue, Mail stop RY-32-2 I I Rahway, NJ 07065, USA

Tel +I 7325942506

Email john.hawkshead.iii@merck.com

Susan E Hassig

Department of Epidemiology, Tulane University School of Public Health and Tropical Medicine, New Orleans, LA, USA

$\mathrm{Tel}+\mathrm{I} 5049886454$

Email shassig@tulane.edu
Background: Pediatric Candida infections are associated with worse clinical outcomes and increased costs. Yet, it is not definitively known if particular species are associated with more severe illness. Differential risk factor exposures among the species group may also exist. We aimed to determine whether certain Candida species are more strongly associated with worse outcomes, and whether certain risk factors more strongly predispose patients to infection with certain species.

Methods: Microbiology lab records from patients seen from 2003 to 2010 at an urban children's hospital were reviewed for invasive or disseminated Candida infections. Data on measures of disease severity/outcome and risk factors were abstracted and analyzed to determine differences associated with various Candida species.

Results: Exactly 106 cases of infection were analyzed. Non-albicans species were associated with a significantly longer length of stay postdiagnosis $(P=0.03)$, as well as longer treatment $(P=0.02)$. Candida albicans was associated with a higher number of antihypotensive medications required $(P=0.03)$ and length of mechanical ventilation postdiagnosis $(P=0.05)$. Candida tropicalis was associated with the highest mortality (45.5\%). Hypotension, which was found to be significantly associated with concurrent infection, was significantly associated with increased risk of mortality (odds ratio $=5.85, P=0.005$ ). Initial choice of antifungal therapy was not associated with differences in eventual patient mortality. Multivariate logistic regression modeling revealed a trend toward C. albicans infection in patients receiving antineoplastic chemotherapy and non-albicans infection in patients with $>96$ hours mechanical ventilation.

Conclusion: Interspecies differences may exist for Candida in terms of disease severity and risk factors. Underlying morbidity and the role of concurrent infections may play a key role in poor outcomes.

Keywords: Candida, disseminated, pediatric, severity, risk factors, fungemia, antifungal, coinfection

\section{Introduction}

Disseminated or invasive Candida infections in pediatric patients, though relatively rare, are associated with significant morbidity and mortality, along with increased societal cost. Mortality in children with invasive candidiasis - that is, infection of the bloodstream or solid organ infection - has been reported from $19 \%$ to $26 \%$ and as high as $43 \%-54 \%$ in infants. ${ }^{1}$ Invasive candidiasis has been associated with a mean 21.1 day increase in length of stay among pediatric patients, in general, incurring a mean increase in total per-patient hospital charges of over $\$ 92,000 .{ }^{2}$ While demonstrably large, the burden of invasive or disseminated Candida infections in pediatric patients 
may still be underappreciated. Current data may reflect underdiagnosis, as investigators have noted that positive prediction of candidemia in the individual patient remains elusive $^{3}$ due to the nonspecificity of symptoms. In adult patients with hematologic malignancies, blood cultures for invasive candidiasis have been found to have a sensitivity only slightly higher than $21 \%$ in select populations, compared to autopsy findings. ${ }^{4}$ Other autopsy-based studies have found slightly better, but still low sensitivity levels of 50\%-60\% for blood cultures in cases of suspected candidemia. ${ }^{2}$ Assuming that blood culture sensitivity is similarly low in pediatric patients, invasive or disseminated Candida infection may impose an even greater burden than is currently appreciated.

Hospitalized pediatric patients are susceptible to invasive or disseminated Candida infections due to neutropenia, extended use of central venous or peripherally inserted catheters, and administration of anti-infective therapy that disturbs the balance of normal endogenous flora, among numerous other risk factors. ${ }^{5}$ In certain intensive care settings such as pediatric and neonatal intensive care units (NICUs), simultaneous exposure to several known risk factors for extended periods of time is common, contributing to patients' susceptibility to these infections.

Besides Candida albicans, other species typically seen in pediatric patients include Candida tropicalis, which is particularly associated with disseminated disease in cancer and leukemia patients with prolonged granulocytopenia. Candida parapsilosis and Candida zeylanoides have been associated with central catheters inserted for extended periods. Candida lusitaniae has been associated with fungemia in immunocompromised patients. ${ }^{6}$ Candida glabrata is also found in pediatric oncology patients, ${ }^{7}$ as is the commonly azole-resistant species Candida krusei. ${ }^{8}$ Taken together, nonalbicans species have been found to constitute the majority of these infections found in this particular patient population in relatively recent studies. ${ }^{9,10}$

In general, although Candida and other fungal pathogens continue to cause significant morbidity and mortality among immunosuppressed pediatric patients, data on invasive fungal infections in pediatric patients, in general, are still underrepresented in the literature. ${ }^{11}$ This is also the case with data on efficacy of available antifungal therapies. ${ }^{5}$ In particular, findings regarding differences in outcome associated with the various Candida species are sparse and inconsistent, and studies analyzing differences in the distribution of various risk factors among a large cohort of pediatric patients infected with different Candida species appear to be lacking as well. This study is intended to enhance our understanding of the course of clinical illness and the risk factors associated with the various Candida species causing invasive or disseminated infections among pediatric patients.

\section{Methods}

The study entailed a retrospective review of paper and electronic records of all patients seen at children's hospital in New Orleans, LA, USA, over a slightly longer than 7-year period (January 2003-April 2010) with a diagnosis of invasive or disseminated Candida infection of the bloodstream or solid organs, confirmed by microbiology culture. Records from the hospital's microbiology laboratory during the relevant period were reviewed to find confirmed cases of invasive or disseminated Candida infection. Data from the hospital's medical records department were used to identify additional cases that may not have been reflected on the microbiology report. As oncology patients are especially susceptible to these infections, the hospital's tumor registry was consulted for a list of patients entered into the registry over the study time frame. This listing was cross-referenced with the microbiology report, and patients' records were reviewed for evidence of bloodstream or deep organ infection. Epidemiology reports from the hospital's infectious disease service were used to identify any further cases that may not have been identified using other methods. Patients eligible for inclusion in the study were those $0-18$ years of age, who developed an invasive or disseminated Candida infection of any species during the course of their inpatient treatment or were transferred to the hospital from other facilities or presented from home with infection. No patients were excluded on the basis of race, ethnicity, socioeconomic status, or referral source. The study was approved by institutional review boards of Tulane University and the Louisiana State University Health Science Center. Written informed patient consent was not obtained.

In addition to demographic and clinical data, the following data were collected for each eligible patient:

- Disease severity/outcome measures, based on clinical markers of invasive Candida infection: ${ }^{12}$ length of hospitalization following diagnosis, in days; time to clear, calculated as the time from initiation of antifungal therapy to the first negative blood culture following initiation of therapy; number and total days of antifungals required; admission to intensive care unit (ICU), and length of ICU stay if admitted; need for mechanical ventilation, and duration of ventilation if needed; deep organ involvement, with specific organ involved; hypotension and number of antihypotensive medications required; and 
fungemia-associated mortality, defined as death within 30 days of diagnosis of candidemia, regardless of whether the infection was successfully treated.

- Risk factors for invasive or disseminated infection, based on significant associations found in previous studies of pediatric patients: $:^{1,4,2,8,9}$ corticosteroid therapy; central venous catheter (CVC) insertion; arterial catheterization; mechanical ventilation; continuous mechanical ventilation for more than 96 hours; total parenteral nutrition begun within 1 month of infection; antineoplastic chemotherapy; prolonged ICU stay; broad-spectrum antibiotic therapy/prophylaxis for more than 1 week; antifungal prophylaxis, with specific antifungal agent(s) used; transfusion within 2 weeks of infection; hemodialysis; recent surgery other than central line placement; and immunosuppression, defined as neutropenia (absolute neutrophil count of $<500$ cells $/ \mu \mathrm{L}$ ), use of immunosuppressant drugs, and/ or history of bone marrow transplantation.

For both disease severity and the risk factor components of this study, patients were assigned to groups based on the species of Candida causing the initial invasive or disseminated infection during the course of treatment.

Statistical tests used to determine whether disease severity differed by species included chi-square and Fisher's exact test analysis, $t$-test, Wilcoxon/Mann-Whitney rank-sum analysis, and Kaplan-Meier survival analysis, performed according to the characteristics of the data and size of groups within organism categories. Stratification for control of confounding factors was based on the demographic and clinical variables specified earlier. Also, Pearson correlation analysis was used to evaluate the effectiveness of treatment of the various fungal species. Species-specific mortality rates according to the first antifungal used after initiation of candidemia therapy were compared, as were the mortality rates according to the numbers of antifungals used after initiation of therapy. Logistic regression with mortality as the outcome and Candida species among the risk factors was also performed to gauge the contribution of fungal infection to patient death. For the risk factor analysis, chi-square, Fisher's exact test, and logistic regression were used for univariate analyses of dichotomous and continuous risk factors, respectively. Based on the results of these analyses, multivariate analysis was accomplished by stepwise logistic regression modeling, using two dichotomous outcome organization scenarios (albicans vs non-albicans, and albicans vs parapsilosis). Again, stratification for control of confounding was based on the demographic and clinical variables specified earlier.
Data analysis was performed using Stata version 12 (StataCorp LP, College Station, TX, USA), MedCalc version 12.2 (Mariakerke, Belgium), and StatCrunch (Pearson Education, College Station, TX, USA) statistical software. Results with a $P$-value $\leq 0.05$ were considered statistically significant for all phases of the study.

\section{Results}

The initial patient roster obtained totaled 265 cases of Candida infection or colonization. Once cases of noninvasive infection or colonization were identified $(n=118)$, patients who were either ineligible due to age above 18 years $(n=8)$, seen outside of the specified time frame $(n=9)$, or whose clinical records were unavailable or ambiguous $(n=17)$ were excluded, and thus, 113 episodes of invasive infection occurring in 106 unique patients remained for full record review and data collection. To eliminate the effects of personal characteristics of patients on the analysis, in instances where an individual patient contracted multiple infections either over the course of a single hospitalization or in more than one hospitalization over time, only that patient's first infection was included. The resultant final dataset comprised 106 cases.

Select demographic characteristics of the study cohort are shown in Table 1. The mean age of patients reviewed was 5.24 years (median age of 1 year, range: 0-18 years, standard deviation 6.79 years). Of the 64 patients with concurrent infection (CI), 41 (64.1\%) had one CI, 12 (18.8\%) had two CIs, and five (7.8\%) had more than two CIs. Among Gram-positive bacterial isolates, the CIs were predominantly Staphylococcus and Enterococcus species; among Gram-negatives, Klebsiella, Pseudomonas, and Escherichia coli were the most frequent findings. Common underlying/ admitting diagnoses included extreme immaturity/preterm birth, necrotizing enterocolitis, short gut syndrome, infection/ inflammatory reaction related to an internal device, sepsis,

Table I Baseline cohort characteristics

\begin{tabular}{ll}
\hline Patient characteristics & $\mathbf{n}(\%)$ \\
\hline Sex & \\
Females & $49(46.2)$ \\
Males & $57(53.8)$ \\
Age & \\
$\quad$ Non-neonate & $58(54.7)$ \\
$\quad$ Neonate & $48(45.3)$ \\
Cancer diagnosis & \\
No & $94(88.7)$ \\
Yes & $12(11.3)$ \\
Concurrent infection & \\
$\quad$ No & $42(39.6)$ \\
Yes & $64(61.9)$ \\
\hline
\end{tabular}


Table 2 Frequency of Candida species identified

\begin{tabular}{lll}
\hline Species & Frequency, $\mathbf{n}$ & $\begin{array}{l}\text { Relative } \\
\text { frequency (\%) }\end{array}$ \\
\hline Candida albicans & $5 \mathrm{I}$ & 48.1 \\
Candida glabrata & 5 & 4.7 \\
Candida parapsilosis & 39 & 36.8 \\
Candida tropicalis & II & 10.4 \\
\hline
\end{tabular}

pneumonia, and respiratory failure. Of the 106 episodes, 105 isolates (99.1\%) were initially isolated from blood cultures. Nine cases (8.5\%) progressed to deep organ involvement (spleen, brain, bone, heart, kidney, retina, and liver).

Species breakdown of invasive/disseminated Candida infections diagnosed at the hospital are shown in Table 2. Changes over time in the annual species distribution are depicted in Figure 1. Annual incidence of invasive infection is depicted in Table 3. Species isolated according to neonatal status are depicted in Figure 2. Binary disease severity measures for the entire cohort are shown in Table 4; continuous disease severity measures are shown in Table 5.

In preliminary analysis, there was a significant association between neonatal status and the Candida species isolated (chi-square $P=0.009$, Fisher's exact test $P=0.007$ ). C. parapsilosis was found to be disproportionately isolated from neonates (24 of 48 isolates [50.0\%]), compared to 15 of 58 isolates $(25.9 \%)$ in the older segment of the cohort. Subsequent analyses of the association between Candida species and disease severity/outcome measures were, therefore, stratified by age. Cancer patients tended to be substantially older than non-cancer patients (median age 6 vs 1 year). Age was possibly significantly associated with the occurrence of deep organ involvement (chi-square $P=0.010$,

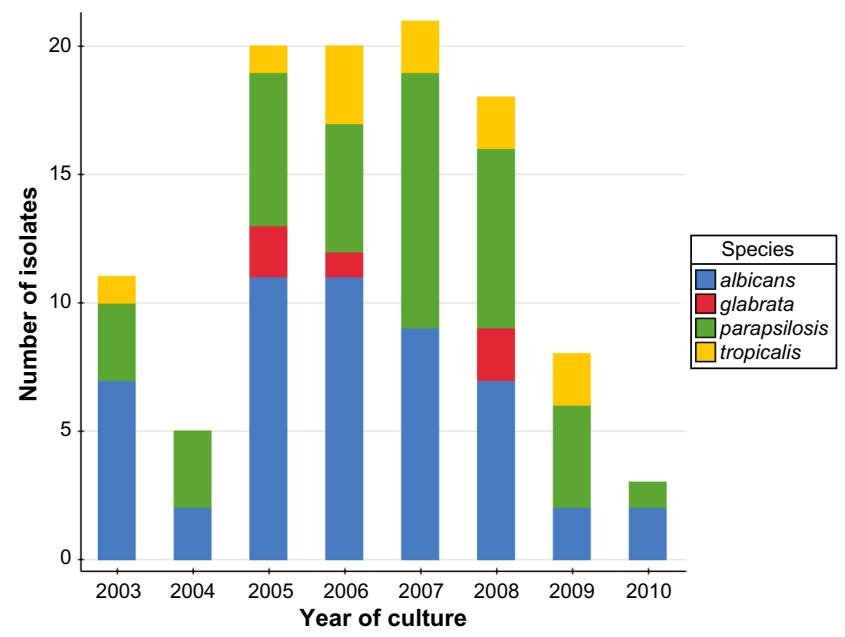

Figure I Change in distribution of Candida species over time. Note: Data for 2010 does not represent the entire year.
Table 3 Candida incidence per I,000 patient days, 2004-20I0

\begin{tabular}{llll}
\hline Year & $\begin{array}{l}\text { Admissions, } \\
\text { n per year }\end{array}$ & $\begin{array}{l}\text { Total Candida } \\
\text { patients, } \\
\text { n per year }\end{array}$ & $\begin{array}{l}\text { Candida incidence, n per } \\
\text { I,000 hospital admissions }\end{array}$ \\
\hline 2004 & 7,916 & 5 & 0.63 \\
2005 & 6,536 & 20 & 3.06 \\
2006 & 6,162 & 20 & 3.25 \\
2007 & 7,226 & 21 & 2.91 \\
2008 & 7,517 & 18 & 2.39 \\
2009 & 8,511 & 8 & 0.94 \\
Total & 43,868 & 92 & 2.10 \\
\hline
\end{tabular}

Note: Full-year admission data for 2003 and 2010 were not available.

Fisher's exact test $P=0.178$ ), which was experienced by the youngest patients more frequently than older patients. CI was strongly associated with greater likelihood of patients being placed on a ventilator within 5 days of invasive Candida diagnosis (chi-square $P=0.006$, Fisher's exact test $P=0.009$ ), being admitted to ICU within 5 days of diagnosis (chi-square $P=0.006$, Fisher's exact test $P=0.009$ ), and developing hypotension (chi-square $P=0.083$, Fisher's exact test $P=0.104)$. CI was associated with a decreased likelihood of deep organ fungal infection (chi-square $P=0.006$, Fisher's exact test $P=0.013$ ).

No significant difference was found between Candida species among the entire study cohort in terms of likelihood of ICU admission or mechanical ventilator placement within 5 days of Candida diagnosis, days to clear the infection, likelihood of hypotension development, likelihood of deep organ involvement, or mortality. Significant interspecies differences were found in several measures in a comparison based on C. albicans versus non-albicans candidiasis,

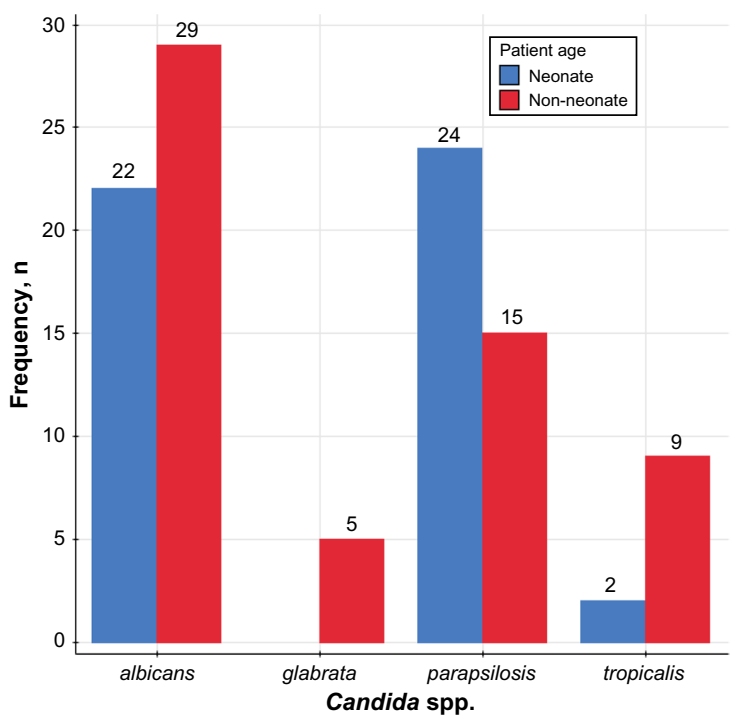

Figure 2 Candida species isolated by patient age-group (neonate, non-neonate). 
Table 4 Binary disease severity measures (entire cohort)

\begin{tabular}{ll}
\hline Measure & $\mathbf{n}(\%)$ \\
\hline ICU admission within 5 days & \\
No & $3(78.3)$ \\
Yes & $23(21.7)$ \\
Ventilator placement within 5 days & \\
$\quad$ No & 8 I (76.4) \\
Yes & $25(23.6)$ \\
Deep organ involvement & \\
No & $96(90.6)$ \\
Yes & $10(9.4)$ \\
Hypotension & \\
No & $65(6 I .3)$ \\
Yes & $4 I(38.7)$ \\
Fungal-related death & \\
No & $78(73.6)$ \\
Yes & $28(26.4)$ \\
\hline Abbreviation: ICU, intensive care unit.
\end{tabular}

such as length of hospitalization postdiagnosis, antifungal days, length of mechanical ventilation, and number of blood pressure raising medications required (Table 6). Non-albicans infection was associated with a longer hospitalization postdiagnosis and number of antifungal days, while C. albicans was associated with a higher number of antihypotensive medications required and the length of mechanical ventilation postdiagnosis.

In subgroup analyses defined by the demographic/clinical variables selected for stratification, no significant interspecies differences were observed among males in any continuous measure of disease severity. Differences in length of hospitalization postdiagnosis between the patient groups defined by species were significant, however, when analysis was limited to females $(P=0.003, C$. albicans vs non-albicans $)$. The difference remained significant when analysis was more narrowly focused on neonate females $(P=0.005$, C. albicans vs non-albicans). In all comparisons, non-albicans species were associated with longer hospitalization. Also, among patients who had a CI, differences in length of hospitalization postdiagnosis remained significantly higher for non-albicans patients $(P=0.045)$. Length of mechanical ventilation was longer in $C$. albicans patients with $\mathrm{CI}$, however $(P=0.041)$, and $C$. albicans patients also typically required more antihypotensive medications ( $P=0.013$ ).

In subgroup analysis performed based on neonatal status, older non-albicans patients experienced significantly longer hospital stay postdiagnosis $(P=0.002, C$. albicans vs $C$. parapsilosis only), while older $C$. albicans patients required more antihypotensive medications $(P=0.048$, C. albicans vs non-albicans). A similar general pattern of interspecies differences was observed when subgroups were defined according to cancer status, which was to be expected given the significant association between age and cancer status (chi-square $P=0.034$, Fisher's exact test $P=0.061$ ).

Interspecies differences in mortality within 30 days of fungemia diagnosis were not significant across the entire cohort. Of all fungal species, C. tropicalis, which proved to typically take longer to clear than other species, was associated with the highest mortality, with five of eleven total patients $(45.5 \%)$ dying within 30 days of confirmation of infection. This total includes two patients whose infections were not confirmed until after they expired. Kaplan-Meier survival analysis showed nearly $65 \%$ survival of $C$. tropicalis patients at 15 days following diagnosis, compared to $86 \%$ survival at a similar point postdiagnosis for C. albicans and C. parapsilosis patients. This difference was not significant, however $(P=0.250)$.

Not all diagnosed cases of candidemia were treated: Eight of the 106 patients in the cohort (7.5\%) did not receive antifungal treatment because cultures taken to confirm the presence of infection were not taken until the patient died, or postmortem. These cases represented four C. albicans infections (median length of hospitalization prediagnosis 6 days), two C. tropicalis infections (4 days), one C. parapsilosis

Table 5 Continuous disease severity measures (entire cohort)

\begin{tabular}{|c|c|c|c|c|}
\hline Measure & $\mathbf{n}$ & $\begin{array}{l}\text { Mean } \\
\text { (SD) }\end{array}$ & $\begin{array}{l}\text { Median } \\
\text { (IQR) }\end{array}$ & $\begin{array}{l}\text { Normality } \\
\text { P-value* }\end{array}$ \\
\hline LOS post Candida diagnosis (days) & 98 & $55.0(70.5)$ & $29.5(15.0,71.0)$ & $<0.0001$ \\
\hline Number of antifungals & 98 & $\mathrm{I} .8(0.7)$ & $2.0(1.0,2.0)$ & 0.001 \\
\hline Antifungal days & 98 & $27.4(27.8)$ & $18.0(13.0,28.0)$ & $<0.0001$ \\
\hline Days to clear & 98 & $10.3(1 \mathrm{I} .2)$ & $6.0(4.0,13.0)$ & $<0.0001$ \\
\hline LOS in ICU (days) & 23 & $14.3(14.5)$ & $10.0(4.0,22.0)$ & 0.001 \\
\hline Length of mechanical ventilation (days) & 25 & $15.2(13.9)$ & $12.0(4.0,19.0)$ & 0.002 \\
\hline Number of BP raising medications & 40 & $\mathrm{I} .6(0.7)$ & $2.0(1.0,2.0)$ & 0.264 \\
\hline Number of transfusions & 66 & $4.7(5.5)$ & $3.0(1.0,6.0)$ & $<0.0001$ \\
\hline
\end{tabular}

Note: *Shapiro-Wilk $W$ test for normality.

Abbreviations: BP, blood pressure; ICU, intensive care unit; IQR, interquartile range; SD, standard deviation; LOS, length of stay. 
Table 6 Analysis of continuous measures of disease severity

\begin{tabular}{|c|c|c|c|c|c|c|c|c|}
\hline \multirow[t]{2}{*}{ Measure } & \multicolumn{3}{|c|}{ Candida albicans } & \multicolumn{3}{|c|}{ Non-albicans } & \multirow{2}{*}{$\begin{array}{l}\text { t-test } \\
P \text {-value }\end{array}$} & \multirow[t]{2}{*}{ P-value* } \\
\hline & $\mathbf{n}$ & Mean (SD) & Median & $\mathbf{n}$ & Mean (SD) & Median & & \\
\hline LOS post Candida diagnosis (days) & 47 & $38.9(40.8)$ & 23.0 & 51 & $69.9(87.5)$ & 51.0 & 0.029 & 0.033 \\
\hline Number of antifungals & 47 & I.7 (0.7) & 2.0 & 51 & I.8 (0.7) & 2.0 & 0.394 & 0.407 \\
\hline Antifungal days & 47 & $22.6(25.2)$ & 15.0 & 51 & $31.8(29.5)$ & 23.0 & 0.101 & 0.023 \\
\hline Days to clear & 47 & $8.1(7.8)$ & 6.0 & 51 & $12.2(13.4)$ & 6.0 & 0.072 & 0.328 \\
\hline LOS in ICU (days) & 10 & 19.6 (18.7) & 12.5 & 13 & $10.2(9.0)$ & 7.0 & 0.127 & 0.384 \\
\hline Length of mechanical ventilation (days) & 12 & $22.0(16.8)$ & 18.0 & 13 & $9.0(6.5)$ & 7.0 & 0.016 & 0.049 \\
\hline Number of BP raising medications & 19 & $1.9(0.7)$ & 2.0 & 21 & I.4 (0.6) & 1.0 & 0.024 & 0.036 \\
\hline Number of transfusions & 32 & $4.0(4.7)$ & 3.0 & 34 & $5.4(6.2)$ & 3.0 & 0.326 & 0.426 \\
\hline
\end{tabular}

Note: *Wilcoxon/Mann-Whitney rank-sum (Mann-Whitney) test.

Abbreviations: BP, blood pressure; ICU, intensive care unit; SD, standard deviation; LOS, length of stay.

infection (70 days), and one C. glabrata infection (6 days). To compare days of treatment with time to clear infection in the 98 instances of treated infections, Pearson correlation analysis with two-sided significance tests was performed for the various Candida species groups. Significant correlations were found between total antifungal days and days to clear infection for the $C$. albicans $(P=0.020), C$. parapsilosis ( $P=0.0001)$, and $C$. tropicalis $(P=0.0001)$ groups. The correlation coefficient for the $C$. glabrata group, however, was not significant $(P=0.908)$, pointing to a possibly unnecessarily long course of treatment of this particular species, although the very small number of patients infected with this species precludes interpretation. C. glabrata otherwise was not significantly associated with more serious outcomes such as mortality, extended length of ICU stay, and mechanical ventilation near the time of candidemia diagnosis.

Across the entire cohort, the overall mortality rate was $20.4 \%$, representing 20 deaths within 30 days of fungemia diagnosis. Rates of mortality in the four groups defined by initial antifungal treatment choice (amphotericin B, fluconazole, voriconazole, and micafungin) did not significantly differ from one another $(P=0.427)$. No significant differences were observed when the cohort was divided into $C$. albicans ( $P=0.400)$ versus non-albicans $(P=0.881)$ groups, males ( $P=0.286)$ versus females $(P=1.000)$, cancer $(P=0.909)$ versus cancer free $(P=0.492)$, or $\mathrm{CI}(P=0.482)$ versus no CI $(P=0.301)$. Similar kinds of variation were not observed when data were stratified according to sex, age, and cancer status. Finally, across the entire cohort and in subgroups, no differences were observed in mortality according to the number of antifungals used.

In a logistic regression model to assess the relationship between infection with albicans versus non-albicans Candida species and the risk of death within 30 days, infection with C. parapsilosis was significantly associated with lower mortality risk within 30 days of diagnosis (odds ratio $[\mathrm{OR}]=0.200, P=0.033$ ). In this model, the presence of hypotension, which previous analysis showed to be significantly associated with CI, was significantly associated with increased risk of mortality $(\mathrm{OR}=5.85, P=0.005)$. A similar association between hypotension and mortality was observed in the C. albicans versus non-albicans regression $(\mathrm{OR}=4.63$, $P=0.007)$. Finally, in the model limited to cases of C. albicans and C. parapsilosis infection, deep organ involvement $(\mathrm{OR}=28.59, P=0.019)$ and prolonged ICU stay $(\mathrm{OR}=14.67$, $P=0.035)$ were, along with hypotension ( $\mathrm{OR}=12.84, P=0.002$ ), associated with significantly increased risk of mortality.

For the risk factor segment of the study, fewer records were available (98 vs 106 for the disease severity/outcome segment). In general, risk factor data were more difficult to collect. For example, patients with infections had transferred to the hospital from other facilities or from home. In such cases, hospital-associated risk factor data were either nonexistent or their documentation was imprecise. For some patients, risk factor data were based on hospital admission notes, and were not from original documentation generated by the patient's previous hospital. Also, in some cases, it was necessary to review data from hospital stays that immediately preceded visits in which patients developed Candida infection, as relevant risk factors associated with the infection (eg, central line insertion and other invasive procedures) may have occurred during the preceding visit. Such a methodology was applied in four cases. In some cases, data for exposure variables included in the analysis could not be reliably determined (Total parenteral nutrition [TPN] [1], antifungal prophylaxis [3], transfusion [1], hemodialysis [2], and immunosuppression [1]), but this was not judged as having potentially significant impact on the results because of the relatively very small numbers of such occurrences in relation to the overall sample size. 
Table 7 Dichotomous risk factor distribution

\begin{tabular}{|c|c|}
\hline Risk factor & n (\%) \\
\hline \multicolumn{2}{|c|}{ Corticosteroid therapy } \\
\hline No & $73(74.5)$ \\
\hline Yes & $25(25.5)$ \\
\hline \multicolumn{2}{|c|}{ CVC insertion } \\
\hline No & $14(14.3)$ \\
\hline Yes & $84(85.7)$ \\
\hline \multicolumn{2}{|c|}{ Recent mechanical ventilation } \\
\hline No & $40(40.8)$ \\
\hline Yes & $58(59.2)$ \\
\hline \multicolumn{2}{|c|}{$>96$ hours mechanical ventilation } \\
\hline No & $4 \mid(4 \mid .8)$ \\
\hline Yes & $57(58.2)$ \\
\hline \multicolumn{2}{|l|}{ TPN } \\
\hline No & $38(39.2)$ \\
\hline Yes & $59(60.8)$ \\
\hline \multicolumn{2}{|c|}{ Antineoplastic chemotherapy } \\
\hline No & $89(90.8)$ \\
\hline Yes & $9(9.2)$ \\
\hline \multicolumn{2}{|c|}{ Prolonged ICU stay } \\
\hline No & $39(39.8)$ \\
\hline Yes & $59(60.2)$ \\
\hline \multicolumn{2}{|c|}{ Antifungal prophylaxis } \\
\hline No & $80(84.2)$ \\
\hline Yes & $15(15.8)$ \\
\hline \multicolumn{2}{|c|}{$>$ I week broad-spectrum antibiotics } \\
\hline No & $17(17.3)$ \\
\hline Yes & $81(82.7)$ \\
\hline \multicolumn{2}{|l|}{ Transfusion } \\
\hline No & $34(35.1)$ \\
\hline Yes & $63(64.9)$ \\
\hline \multicolumn{2}{|l|}{ Hemodialysis } \\
\hline No & $90(93.8)$ \\
\hline Yes & $6(6.3)$ \\
\hline \multicolumn{2}{|l|}{ Surgery } \\
\hline No & $60(6 \mid .2)$ \\
\hline Yes & $38(38.8)$ \\
\hline \multicolumn{2}{|c|}{ Immunosuppression } \\
\hline No & $72(74.2)$ \\
\hline Yes & $25(25.8)$ \\
\hline
\end{tabular}

Abbreviations: ICU, intensive care unit; CVC, central venous catheter; TPN, total parenteral nutrition; CVC, central venous catheter.

Distribution of dichotomous risk factor exposures for the cohort at large is shown in Table 7. No significant differences were found in exposure to the following defined risk factors among species groups, both in analysis of the entire cohort and stratified according to demographic and clinical characteristics: corticosteroid therapy, central venous catheter (CVC) insertion, mechanical ventilation within 2 weeks prior to diagnosis, total parenteral nutrition, transfusion within 2 weeks, exposure to broad-spectrum antibiotics for more than 1 week, and hemodialysis. As was the case with disease severity analysis, several significant differences in risk factor exposure among Candida species groups and subgroups within those categories were found. Recent exposure to long-term mechanical ventilation (more than 96 continuous hours) was associated with a greater likelihood of developing non-albicans fungemia in C. albicans versus non-albicans analysis (chi-square $P=0.029$, Fisher's exact test $P=0.040$ ). This association was as significant when the analysis was limited to $C$. albicans and C. parapsilosis patients (chi-square $P=0.026$, Fisher's exact test $P=0.042$ ). The association was particularly strong when one CI was present (chi-square $P=0.002$, Fisher's exact test $P=0.004$ ). Antineoplastic chemotherapy exposure was associated with a significantly greater risk of $C$. albicans than non-albicans fungemia (chi-square $P=0.010$, Fisher's exact test $P=0.013$ ). Prolonged ICU stay, such as that often experienced by neonates, was significantly associated with greater likelihood of $C$. parapsilosis infection (chi-square $P=0.026$, Fisher's exact test $P=0.037$ ) than C. albicans infection. Nearly $80.6 \%$ of $C$. parapsilosis cases were preceded by an extended ICU stay, compared to $51.0 \%$ of $C$. albicans cases.

Antifungal prophylaxis was not significantly associated with increased risk of non-albicans fungemia (chi-square $P=0.203$, Fisher's exact test $P=0.264$ ) in the cohort at large. When the analysis was limited to the non-oncology patient population, prophylaxis was associated with moderately increased risk of $C$. parapsilosis infection, but this association did not reach statistical significance (chi-square $P=0.062$ ). Finally, in regard to the impact of days of hospitalization on the fungal species isolated, univariate logistic regression analysis showed that length of hospitalization prior to diagnosis was moderately associated with greater likelihood of development of non-albicans fungemia $(\mathrm{OR}=1.018, P=0.057)$. This result reflects the prevalence of $C$. parapsilosis infection among the sizeable neonatal segment of the study population and the extended prediagnosis lengths of stay commonly found in this subset of the population. Another risk factor with possibly differential Candida species associations was immunosuppression, which was found to be possibly associated with slightly higher risk of $C$. albicans and C. tropicalis infection in older females (chi-square $P=0.053$ ).

Following univariate analyses, logistic regression modeling was performed, with C. albicans/non-albicans and C. albicans/C. parapsilosis as the defined outcome groups and the following variables as predictors: sex, age, CI, antineoplastic chemotherapy, prolonged ICU stay, antifungal prophylaxis, broad-spectrum antibiotic exposure for more than 1 week, recent surgery, continuous mechanical ventilation for more than 96 hours, transfusion, and immunosuppression. Second models for both outcome scenarios were then run 
based on numbers of procedures and transfusions. In the C. albicans/non-albicans models, the only risk factors found to be nearly significantly associated with differential risk of a particular Candida species group were antineoplastic chemotherapy with $C$. albicans $(\mathrm{OR}=12.50, P=0.058)$ in the first model and $>96$ hours mechanical ventilation with nonalbicans $(\mathrm{OR}=6.17, P=0.059)$ in the second model.

\section{Discussion}

Regarding the measures of disease severity and ultimate outcomes, this study found that some observed differences between Candida species might be, in part, related to the high prevalence of $C$. parapsilosis among very young patients (49.0\% vs $26.6 \%$ in older patients), a segment of the population characterized by extended hospital stays and preponderance of risk factors such as long-term mechanical ventilation, CVCs, both antibacterial and antifungal prophylaxis, gastrointestinal (GI) difficulties such as short gut syndrome and necrotizing enterocolitis, and concurrent bacterial or viral infection. Bacterial coinfection has been found to deplete immunity and predispose patients, especially those having low birth weight or congenital anomalies or both, to secondary invasive Candida infection, while use of broadspectrum antibiotics, especially when administered for longer than 1 week (as was the case with nearly $83 \%$ of patients in this study), has been associated with elimination of protective bacterial flora in the gut and on other mucosal surfaces, Candida colonization, and increased infection risk. ${ }^{13-15}$ Further, invasive devices such as endotracheal tubes and venous access devices allow increased likelihood of colonization of a patient's internal spaces with both fungal and bacterial organisms from endogenous or exogenous sources that can then more easily multiply and cause severe infection. In this cohort, concurrent Candida and other infections may have acted in concert to produce hypotension and, thus, increased mortality among both $C$. parapsilosis $(P=0.031)$ and especially $C$. albicans patients $(P=0.0001)$. In general, patients with $C$. albicans, females in particular, were more prone to mortality within 30 days than $C$. parapsilosis patients, possibly due to $C$. albicans' association with predictors of poor outcome, such as significantly more severe hypotension, as measured by the number of antihypotensive medications required, and longer duration of mechanical ventilation postdiagnosis than non-albicans species. Other investigators ${ }^{16,17}$ have noted an apparently stronger association in pediatric patients between $C$. albicans infection and death than nonalbicans species, $C$. parapsilosis in particular.

Another finding of this study that affirms previous research ${ }^{18-20}$ is the high mortality associated with $C$. tropicalis infection, which, at $38.5 \%$ of cases, was significantly higher than the rates associated with the other three species isolated among the study population. Patients with $C$. tropicalis infections tended to die more quickly than patients infected with other species, perhaps owing to the longer time it took for the infection to clear compared to other species. C. tropicalis appears to display higher potential for dissemination in immunocompromised hosts than C. albicans and other nonalbicans species, ${ }^{21}$ possibly in part explaining this phenomenon. Furthermore, C. tropicalis patients were noted in several cases to have serious underlying conditions which may have negatively affected their chances for survival. These included cystic fibrosis, trisomy 21 with respiratory distress, Ewing's sarcoma, and peripheral nerve sheath tumor. Initial antifungal therapy for these infections was typically fluconazole, which has been found to have decreasing effectiveness against this species in several studies. ${ }^{19,22,23}$ Also, amphotericin B, which was also deployed for C. tropicalis infections in the cohort, has been noted to be less effective against this species than it previously had been. ${ }^{8}$

Another dimension of candidemia-related mortality encountered in this study was several cases of possibly unrecognized and untreated infection that ultimately proved fatal. In clinical practice, recognition and treatment of fungemia may be delayed because of the nonspecificity of symptoms, delays in reporting of blood culture results from the laboratory, lack of sensitivity of blood cultures for diagnosing deep organ invasion, and individual clinician or institutional practice patterns. ${ }^{24}$ Many clinicians will only start antifungal therapy when a positive culture is reported or when the patient fails to improve after a period of treatment with antibacterial agents. A policy of starting empiric antifungal therapy immediately after obtaining cultures in neonates with risk factors for fungal sepsis, instead of waiting for culture results, could aid in preventing mortality (although such a policy would have to be balanced against the risk of development of antifungal resistance and side effects associated with the agents). The risk factors that the authors recommend to be considered in this scenario include central venous catheterization, mechanical ventilation, time spent in intensive care, and prior broad-spectrum antibiotic exposure, among others. ${ }^{24}$ In this study, seven such cases were encountered, representing one infection with C. glabrata, three with $C$. albicans, two with $C$. tropicalis, and one with C. parapsilosis. All of these patients died.

Among the 13 oncology patients included in this study, C. albicans was the predominant species isolated (eight of $13,61.5 \%$ ). Other investigators have reported that nonalbicans species typically constitute the majority of Candida 
infections in this segment of the pediatric population ${ }^{9,10}$; others, however, have found an even division between albicans and non-albicans species in these patients. ${ }^{8}$ Overall, distributions of species appear to be somewhat specific to individual institutions and departments, depending on the patient population served, treatment and prophylaxis protocols and practices, and infection control measures.

C. albicans was also found to be quite common in the youngest segment of patients studied, accounting for 23 of $49(46.9 \%)$ total cases among neonate patients, just below the $48.9 \%$ accounted for by C. parapsilosis. Despite the latter's prevalence, $C$. albicans has previously been found to be a major infecting species in neonatal settings, both in the USA and internationally. C. albicans was the dominant species isolated in a prospective study conducted in the 1990s, before a shift occurred toward C. parapsilosis. ${ }^{25}$ Another European study conducted in the 1990s found that greater than $83 \%$ of cases of fungemia in neonates were attributable to C. albicans. ${ }^{8}$ Data obtained from the National Nosocomial Infections Surveillance and National Healthcare Safety Network programs over the 1999-2009 period found a nearly exact split between albicans and non-albicans (706 vs 701 cases) among neonatal candidemias. ${ }^{26}$ Results obtained in the current study, showing a large degree of C. albicans infection among this vulnerable patient population, are consistent with that experience. C. albicans is the species most frequently isolated from the neonatal GI tract, and has been found to have greater adherence to neonatal enterocytes compared with other species, leading to penetration and invasion of host cell barriers, and finally dissemination via the bloodstream. ${ }^{27}$ Given the prevalence of GI tract issues among this population, a high prevalence of $C$. albicans fungemia is, therefore, to be expected.

In addition to the risk of infection imposed by patients' underlying illnesses and invasive treatment, Candida infection imposes challenges to clinicians through innate qualities. These include intrinsic or acquired resistance to antifungal therapy, environmental persistence, inadequate infection control practices, and ability to form biofilms on surfaces of medical devices, as noted earlier. Broad use of antifungal agents, particularly fluconazole for prophylaxis, has led to increased concern about the emergence of resistance to this class of drug. ${ }^{22}$ As a result, antifungal resistance surveillance with a particular focus on Candida and azole antifungals has become widespread. In general, resistance to fluconazole has remained consistently low for most of the commonly encountered species in clinical practice. Among greater than 13,300 Candida isolates associated with bloodstream infection that were tested at the University of Iowa from 1998 to 2004, resistance to fluconazole, defined as a minimum inhibitory concentration of greater than $64 \mu \mathrm{g} / \mathrm{mL}$, was less than $3 \%$ for all species, with the exceptions of C. glabrata $(9 \%)$ and especially the highly resistant species C. krusei $(40 \%){ }^{22}$ Accordingly, fluconazole prophylaxis has been found to be an independent risk factor for candidemia caused by these species in recent research, ${ }^{28}$ associated with ORs of 2.04 (95\% confidence interval 1.36-3.06) for disseminated C. glabrata infection and 5.26 (95\% confidence interval 2.92-9.47) for disseminated C. krusei infection. Antifungal resistance was not evident in this study, however. C. glabrata, which has shown reduced susceptibility to azole antifungals, ${ }^{11}$ was associated with the shortest average time to clear of the four Candida species isolated. Initial therapy for the four isolates of C. glabrata that were treated consisted of fluconazole in two cases and amphotericin B in the other two.

In general, targeted fluconazole prophylaxis has been found to be an effective means of preventing $C$. parapsilosis in hospital settings, NICUs in particular. In such units, fluconazole prophylaxis for infants weighing below $1 \mathrm{~kg}$, or for those born after 27 weeks or shorter gestation, is broadly accepted. ${ }^{29}$ Among the youngest segment of the children's hospital cohort, administration of antifungals prior to identification of disseminated Candida infection (either for prophylaxis or for treatment of prior noninvasive fungal infections) did not significantly predispose patients to infection with any particular species in a four-way analysis. It was observed that prior prophylactic or therapeutic antifungal administration was, as noted earlier, associated with a greater risk of C. parapsilosis infection among non-oncology patients in the study ( $P=0.031)$. Such a finding is somewhat unusual, as resistance to fluconazole on the part of $C$. parapsilosis has consistently been found to be rather low, typically ranging from $2 \%$ to $4.2 \%$ among the isolates detected from 1997 to 2003 by the ARTEMIS DISK Antifungal Surveillance Program, in which species identification and antifungal susceptibility profiles of over 134,000 isolates from 39 countries were included. ${ }^{22}$ Elevated levels of fluconazole resistance in C. parapsilosis isolates obtained from NICU patients have been documented in the literature. ${ }^{30}$ Still, it is unclear whether the findings of the current study are due, in greater part, to especially resistant strains of C. parapsilosis in this particular hospital, or to more intensive exposure to one or more risk factors such as length of hospitalization, especially in an NICU setting, and mechanical ventilation. In the absence of molecular characterization of individual isolates in the laboratory, which is not routinely performed, this question cannot be answered satisfactorily. It bears noting that a recent study of antifungal susceptibility of various Candida species conducted in Europe 
found that $70.3 \%$ of $C$. parapsilosis isolates were susceptible to fluconazole, ${ }^{23}$ which means that nearly three out of ten isolates were not susceptible. If trends in evolution of antifungal resistance among this species at the study hospital are similar to those of the European study cited, it is possible that some of the burden of $C$. parapsilosis infection is at least, in part, attributable to more resistant strains being selected out by administration of fluconazole or another antifungal. Nearly $21 \%$ of patients aged less than 1 year (nine of 43 total patients) received antifungal agents prior to onset of fungemia, compared to $11.5 \%$ of patients of age 1 year or above, which was a nonsignificant difference $(P=0.212)$. Schmalreck et $\mathrm{al}^{23}$ have recently noted that non-albicans species are more frequently found to have decreased antifungal susceptibility of late, one of which the authors single out is $C$. parapsilosis.

Echinocandin prophylaxis, identified as a possible risk factor for $C$. parapsilosis infection, ${ }^{29}$ was too infrequently used at children's hospital during the study period to be evaluated, however. Also, isolation of C. glabrata, the species found most commonly associated with breakthrough fungemia, was too rarely found in this study to reliably determine whether its presence was due to prior administration of antifungal agents.

Limitations of the study include it being reflective only of the experience of a single institution with what is still a somewhat rare outcome over a fairly short time frame, so results may not be applicable to other institutions with different patient populations, staffing levels, physician practice patterns, and so on. Also, as is suggested by changes observed in antifungal susceptibility over time as cited earlier, changes in microbiological characteristics of the organisms in question during the study period and beyond may limit the applicability of the findings to present-day settings in different geographic locations. This variability seems likely to become only greater as the length of time beyond the end of the study period and the present time grows longer. Another limitation of the current study is that it includes cases of infection only and does not include a sample of controls against whom to compare disease severity and outcome measures and risk factor exposures. As it is, all patients in the cohort have the outcome of interest, that is, invasive/disseminated Candida infection, so the population is by definition limited to those patients with increased susceptibility to the outcome. The study population, therefore, is not as diverse and generalizable in terms of findings to the larger pediatric population. Given the way the study was designed, with the goal of defining interspecies differences in disease severity/outcome and risk factor exposures, it cannot be ascertained if otherwise comparable patients at the hospital experience similar short- and intermediate-term consequences of their hospitalization, or similar outcomes, to those experienced by Candida patients. Therefore, the precise role of Candida infection in terms of patient acuity and outcome cannot be precisely quantified.

Also, this study did not characterize the experience of patients with noninvasive Candida infections such as urinary tract infections or superficial skin infections, although these patients were identified by microbiology lab records. Such patients would have made a useful contribution to the study population, as prior Candida colonization has been shown to be a significant risk factor for invasive/disseminated infection. Inclusion of these patients would have contributed to the statistical power of the study and helped elucidate the overall contribution of fungal infection to disease severity and outcomes as well as the contribution of identified risk factors to the overall risk of Candida infection in this population. They were not included, however, due to study resource limitations. The study did neither assess the impact of deficiencies and improvements in the hospital's infection control and environmental hygiene programs on the epidemiology of Candida infections in affected patient care areas, nor did it take into account the possible impact of suboptimal medication dosing, which has been shown to be critical in pediatric applications especially. ${ }^{31}$ Other limitations include the aforementioned lack of laboratory characterization of drug-resistant strains and the lack of consideration of socioeconomic and other factors that could have an impact on patients' risks for invasive infection and disease outcomes, for example, substandard catheter care in the home setting. Finally, the study did not consider the effect of CVC removal on outcomes such as length of hospitalization, treatment duration, or mortality. Failure to remove CVCs has been found to be an independent risk factor for early death among pediatric patients with candidemia. ${ }^{32}$ Catheter removal, however, may not always be feasible in children because of potential problems associated with reinsertion and limited vascular access sites. ${ }^{33}$ Retained fixed catheter fragments have also been reported following attempted catheter removal. ${ }^{34}$

Finally, another limitation of this study was the inability to evaluate differences in virulence of the pathogens themselves which might result from innate microbiological characteristics such as their ability to produce biofilms and adhere to and invade host tissues. The former characteristic has been linked to increased risk of eventual patient mortality 
in a recent study, possibly by resultant reduced antifungal effectiveness. ${ }^{35}$

\section{Conclusion}

Results of the present study support the conclusion that critically ill pediatric patients, neonates, and oncology patients, in particular, exposed to long-term hospitalization and its attendant risk factors are susceptible to invasive Candida infections. In general, non-albicans infection was associated with longer hospitalization postdiagnosis and number of antifungal days, while $C$. albicans was associated with a higher number of antihypotensive medications required and length of mechanical ventilation postdiagnosis. C. tropicalis was associated with noticeably higher mortality than other species, but this was not significant, likely due to small sample size. There were no significant differences in mortality outcomes among the various antifungal therapies administered. The underlying medical conditions, CIs, hypotension, and deep organ involvement present among many members of this complex patient population conceivably contributed to poor outcomes and differences in clinical course among the comparison groups. In terms of differential risk factor exposure, long-term mechanical ventilation and prolonged ICU stay (variables that are highly collinear) were associated with non-albicans fungemia, particularly C. parapsilosis, while antineoplastic chemotherapy in older patients was associated with greater risk of $C$. albicans infection in univariate models. Associations were not significant in multivariate analysis.

In conclusion, the clinical and social impact of invasive Candida infections in the pediatric patient population, especially in a time of increasing antifungal resistance and slowed development of new and more effective antifungal agents, argues in favor of the promotion of more coordinated, collaborative research into optimal treatment, prophylactic, and infection prevention strategies.

\section{Acknowledgments}

The authors would like to acknowledge the invaluable assistance of Drs Bhumi Patel and Tanzina Nashid in abstracting hospital chart data for this study.

This work was supported by funding from the Merck Investigator Initiated Studies Program (grant no 36660). The abstract of this paper was presented at the Society for Healthcare Epidemiology of America Spring 2015 Conference, May 14-17, 2015, Orlando, FL, USA, as a poster presentation (conference abstract no 6526).

\section{Disclosure}

The authors report no conflicts of interest in this work.

\section{References}

1. Zaoutis TE, Greves HM, Lautenbach E, Bilker WB, Coffin SE. Risk factors for disseminated candidiasis in children with cancer. Pediatr Infect Dis J. 2004;23:635-641.

2. Zaoutis TE, Argon J, Chu J, Berlin JA, Walsh TJ, Feudtner C. The epidemiology and attributable outcomes of candidemia in adults and children hospitalized in the United States: a propensity analysis. Clin Infect Dis. 2005;41:1232-1239.

3. Glöckner A, Karthaus M. Current aspects of invasive candidiasis and aspergillosis in adult intensive care patients. Mycoses. 2011;54: 420-433.

4. Rosen GP, Nielsen K, Glenn S, Abelson J, Deville J, Moore TB. Invasive fungal infections in pediatric oncology patients: 11 year experience at a single institution. J Pediatr Hematol Oncol. 2005;27:135-140.

5. Queiroz-Telles F, Berezin E, Leverger G, et al; Micafungin Invasive Candidiasis Study Group. Micafungin versus liposomal amphotericin B for pediatric patients with invasive candidiasis: substudy of a randomized double-blind trial. Pediatr Infect Dis J. 2008;27:820-826.

6. Kern ME, Blevins KS, editors. Medical Mycology: A Self-Instructional Text. 2nd ed. Philadelphia: F.A. Davis Company; 1997.

7. Wingard JR. Importance of Candida species other than C. albicans as pathogens in oncology patients. Clin Infect Dis. 1995;20:115-125.

8. Krcmery V, Laho L, Huttova M, et al. Aetiology, antifungal susceptibility, risk factors and outcome in 201 fungaemic children: data from a 12-year retrospective study from Slovakia. J Med Microbiol. 2002;51: $110-116$.

9. Krupova Y, Sejnova D, Dzatkova J, et al. Prospective study on fungemia in children with cancer: analysis of 35 cases and comparison with 130 fungemias in adults. Support Care Cancer. 2000;8:427-430.

10. Mullen CA, El-Baki HA, Samir H, Tarand JJ, Rolston KV. Non-albicans Candida is the most common cause of candidemia in pediatric cancer patients. Support Care Cancer. 2003;11:321-325.

11. Steinbach WJ. Epidemiology of invasive fungal infections in neonates and children. Clin Microbiol Infect. 2010;16:1321-1327.

12. Mayhall CG, editor. Hospital Epidemiology and Infection Control. 2nd ed. Philadelphia: Lippincott Williams \& Wilkins; 1999.

13. Chiu NC, Chung YF, Huang FY. Pediatric nosocomial fungal infections. Southeast Asian J Trop Med Public Health. 1997;28:191-195.

14. Abelson JA, Moore T, Bruckner D, Deville J, Nielsen K. Frequency of fungemia in hospitalized pediatric inpatients over 11 years at a tertiary care institution. Pediatrics. 2005;116:61-67.

15. al Soub H, Estinoso W. Hospital-acquired candidaemia: experience from a developing country. J Hosp Infect. 1997;35:141-147.

16. Faix RG. Invasive neonatal candidiasis: comparison of albicans and parapsilosis infection. Pediatr Infect Dis J. 1992;11:88-93.

17. Zaoutis TE. Candidemia in children. Current Med Res Opinion. 2010;26;1761-1768.

18. Krcmery V, Barnes AJ. Non-albicans Candida spp. causing fungaemia: pathogenicity and antifungal resistance. $J$ Hosp Infection. 2002;50: 243-260.

19. Pappas PG, Rex JH, Lee J, et al. A prospective observational study of candidemia: epidemiology, therapy, and influences on mortality in hospitalized adult and pediatric patients. Clin Infect Dis. 2003; 37:634-643.

20. Singhi SC, Reddy TC, Chakrabarti A. Candidemia in a pediatric intensive care unit. Pediatr Crit Care Med. 2004;5:369-374.

21. Negri M, Silva S, Henriques M, Oliveira R. Insights into Candida tropicalis nosocomial infections and virulence factors. Eur J Clin Microbiol Infect Dis. 2012;31:1399-1412.

22. Pfaller MA, Diekema DJ. Epidemiology of invasive candidiasis: a persistent public health problem. Clin Microbiol Rev. 2007;20:133-163. 
23. Schmalreck AF, Willinger B, Haase G, et al. Species and susceptibility distribution of 1062 clinical yeast isolates to azoles, echinocandins, flucytosine and amphotericin B from a multi-centre study. Mycoses. 2012;55:e124-e137.

24. Cahan H, Deville JG. Outcomes of neonatal candidiasis: the impact of delayed initiation of antifungal therapy. Int J Pediatrics. 2011;2011:813871. doi:10.1155/2011/813871. Epub 2011 Nov 3.

25. Roilides E, Farmaki E, Evdoridou J, et al. Neonatal candidiasis: analysis of epidemiology, drug susceptibility, and molecular typing of causative isolates. Eur J Clin Microbiol Infect Dis. 2004;23:745-750.

26. Chitnis AS, Magill SS, Edwards JR, Chiller TM, Fridkin SK, Lessa FC. Trends in Candida central line-associated bloodstream infections among NICUs,1999-2009. Pediatrics. 2012;130:e46-e52.

27. Falgier C, Kegley S, Podgorski H, et al. Candida species differ in their interactions with immature human gastrointestinal epithelial cells. Pediatr Res. 2011;69:384-389.

28. Hachem R, Hanna H, Kontoyiannis D, Jiang Y, Raad I. The changing epidemiology of invasive candidiasis: Candida glabrata and Candida krusei as the leading causes of candidemia in hematologic malignancy. Cancer. 2008;112:2493-2499.
29. Trofa D, Gácser A, Nosanchuk JD. Candida parapsilosis, an emerging fungal pathogen. Clin Microbiol Rev. 2008;21:606-625.

30. Sarvikivi E, Lyytikainen O, Soll, et al. Emergence of fluconazole resistance in a Candida parapsilosis strain that caused infection in a neonatal intensive care unit. J Clin Microbiol. 2005;43:2729-2735.

31. Steinbach WJ. Critical importance of antifungal dosing in children. Expert Rev Anti Infect Ther. 2011;9:283-284.

32. Pasqualotto AC, de Moraes AB, Zanini RR, Severo LC. Analysis of independent risk factors for death among pediatric patients with candidemia and a central venous catheter in place. Infect Control Hosp Epidemiol. 2007;2:799-804.

33. Shah SS, Smith MJ, Zaoutis TE. Device-related infections in children. Pediatr Clin N Am. 2005;52:1189-1208.

34. Jones SA, Giacomantonio M. A complication associated with central line removal in the pediatric population: retained fixed catheter fragments. J Pediatr Surg. 2003;38:594-596.

35. Rajendran R, Sherry L, Nile CJ, et al. Biofilm is a risk factor for mortality in patients with Candida albicans bloodstream infection Scotland, 2012-2013. Clin Microbiol Infect. 2016;22:87-93.
Infection and Drug Resistance

\section{Publish your work in this journal}

Infection and Drug Resistance is an international, peer-reviewed openaccess journal that focuses on the optimal treatment of infection (bacterial, fungal and viral) and the development and institution of preventive strategies to minimize the development and spread of resistance. The journal is specifically concerned with the epidemiology of antibiotic

\section{Dovepress}

resistance and the mechanisms of resistance development and diffusion in both hospitals and the community. The manuscript management system is completely online and includes a very quick and fair peerreview system, which is all easy to use. Visit http://www.dovepress.com/ testimonials.php to read real quotes from published authors. 